\title{
CALIBRATION RELATIONS FOR ANALOGUES OF THE BASIS SPLINES WITH UNIFORM NODES ${ }^{1}$
}

\author{
Valerii T. Shevaldin

\begin{abstract}
N.N. Krasovskii Institute of Mathematics and Mechanics, Ural Branch of the Russian Academy of Sciences; Ural Federal University, Ekaterinburg, Russia, Valerii.Shevaldin@imm.uran.ru
\end{abstract}

\begin{abstract}
The paper deals with generalized linear and parabolic $B$-splines with the uniform nodes constructed by means only one function $\varphi(x)$. For such splines in this paper conditions have been found that guarantee satisfaction of two-scale relations.
\end{abstract}

Key words: $B$-spline, uniform nodes, two-scale relations.

\section{Introduction}

In contemporary mathematics, various generalizations of the polynomial spline-functions regularly appear. Besides the well-known $\mathcal{L}$-splines (see, for example [1]), let us note the sourcerepresentative splines [2], the Rvachev functions [3], the Leontiev splines [4], the Kvasov isogeometric splines [5], the Demyanovich $B_{\varphi}$-splines [6], and so on.

Recently, the Author [7] suggested another generalization of a known construction of the parabolic basis spline (of the $B$-spline) with the uniform nodes; this spline is constructed by means of only one function $\varphi \in C^{1}[-h, h](h>0)$.

In [7], the approximative and form-retaining properties of the local non-interpolating splines were investigated. These ones are linear combinations of shifts of the suggested $B$-splines. As particular cases, there were considered examples of exponential, elliptic, and hyperbolic local splines with arbitrary collocation of nodes.

It is well known that the polynomial splines have played important role in development of the wavelet theory (see, e.g., [8-10]). Namely, in constructing the wavelet decompositions of the space $L^{2}(\mathbb{R})$, embeddedness of spaces $\left\{V_{j}\right\}_{j=-\infty}^{\infty}$ on refining meshes is used. This embeddedness follows from presence of scaling (multiple-scaled) relations (see $[8, \S 4.3])$ ) for the basis functions.

But note that not each basis function $B(x)$ satisfies the general scaling equation of the form

$$
B(x)=\sum_{j \in \mathbb{Z}} h_{j} B(2 x-j h) \quad(x \in \mathbb{R}),
$$

and finding such functions $B(x)$ is a complicated problem.

In the present paper, conditions on the function $\varphi$ are given that guarantee implementation of analogues for the two-scaled relations for the generalized parabolic $B$-splines from work [7] (all necessary definitions are given below). Moreover, an analogous problem is considered for the generalized linear $B$-splines and corresponding examples are given.

It is worthy to note that we have obtained these results without application of the harmonic analysis techniques.

\footnotetext{
${ }^{1}$ The paper was originally published in Trudy Institute of Mathematics and Mechanics, Ural Branch of the Russian Academy of Sciences, 2011. Vol. 17, no 3. P. 319-323 (in Russian).
} 


\section{Generalized parabolic $B$-splines}

Let $h>0$ and $C=C[a, b]$ be the space of continuous functions given on the segment $[a, b]$ with usual definition of the norm

$$
\|f\|_{C}=\max _{x \in[a, b]}|f(x)| .
$$

Fix the function $\varphi$ given on the segment $[-2 h, 2 h]$ and satisfying the following conditions:

$$
\varphi^{\prime} \in C[-2 h, 2 h], \quad \varphi(-x)=\varphi(x) \quad(x \in[0,2 h]), \quad \varphi(0)=\varphi^{\prime}(0)=0 .
$$

The $B$-spline corresponding to this function $\varphi$ (see [7]) is described by the formula

$$
B_{h, 2}(x)=m(h)\left\{\begin{array}{cl}
\varphi(x), & x \in[0, h], \\
2 \varphi(h)-\varphi(x-h)-\varphi(x-2 h), & x \in[h, 2 h], \\
\varphi(3 h-x), & x \in[2 h, 3 h], \\
0, & x \notin[0,3 h] .
\end{array}\right.
$$

Here, $m=m(h)>0$ is the normalizing multiplier.

In the classic case, the normalized parabolic $B$-spline with the uniform nodes $0, h, 2 h 3 h$ (see [11]) is obtained from this definition if to set $\varphi(x)=x^{2}$ and $m(h)=1 /\left(2 h^{2}\right)$.

Note evident properties of the function $B_{h, 2}(x)$ that follow from conditions (1.1):

$$
\operatorname{supp} B_{h, 2}(x)=[0,3 h], \quad B_{h, 2}^{\prime} \in C(\mathbb{R}), \quad B_{h, 2}(3 h-x)=B_{h, 2}(x),
$$

(i.e., the function $B_{h, 2}$ is even w.r.t. the middle carrier point $x=(3 h) / 2$ ). If to suppose another condition to be satisfied that the function $\varphi(x)$ does not decrease on $[0, h]$, then the graph of $B_{h, 2}(x)$ will have the form of a symmetric "cap" (w.r.t. the point $x=(3 h) / 2$ ) as a the parabolic $B$-spline with the uniform nodes.

In [7], for such functions $\varphi$, the local splines of the following form were investigated

$$
S(x)=S(f, x)=\sum_{j \in \mathbb{Z}} y_{j} B_{h, 2}\left(x+\frac{3 h}{2}-j h\right),
$$

where $y_{j}=f(j h), f: \mathbb{R} \rightarrow \mathbb{R}$. It has been proved that these splines locally satisfy to the property of retaining the original data $y_{j}$ (of the 1-monotonicity type) in the following sense: if $y_{l-1} \leq y_{l} \leq$ $y_{l+1}(l \in \mathbb{Z})$, then the spline $S(x)$ does not decrease on the segment $[(l-1 / 2) h,(l+1 / 2) h](l \in \mathbb{Z})$.

Together with the function $B_{h, 2}(x)$, consider the function

$$
B_{2 h, 2}(x)=m(2 h)\left\{\begin{array}{cl}
\varphi(x), & x \in[0,2 h], \\
2 \varphi(2 h)-\varphi(x-2 h)-\varphi(x-4 h), & x \in[2 h, 4 h], \\
\varphi(6 h-x), & x \in[4 h, 6 h], \\
0, & x \notin[0,6 h]
\end{array}\right.
$$

that is obtained from he function $B_{h, 2}$ by formal substitution of the parameter $h$ by the $2 h$ one. It is evident that, in a general case, the graph of this function can not be obtained from the graph of the function $B_{h, 2}$ by two-times extension along the horizontal axis as it happened un the classic polynomial case (see $[8, \S 4.3]$ ). This is since nowhere the demand of homogeneity property of the function $\varphi$ is imposed. But this is the key reasoning in the described constructing. So, in the subsequent investigation of the wavelets on the basis of these basis functions, the embeddedness 
of corresponding subspaces $\left\{V_{j}\right\}_{j=-\infty}^{\infty}$ on the refining meshes must be understood in some other sense.

In this paper, we are searching for an answer on the following question. For what functions $\varphi$ satisfying conditions (1.1), there exist real numbers $A_{1}, A_{2}, A_{3}$, and $A_{4}$ such that for any $x \in \mathbb{R}$ the equality holds

$$
B_{2 h}(x)=A_{1} B_{h, 2}(x)+A_{2} B_{h, 2}(x-h)+A_{3} B_{h, 2}(x-2 h)+A_{4} B_{h, 2}(x-3 h) ?
$$

We call this equation the scaling (two-scaled) relation for the generalized $B$-spline that is determined by formula (1.2). In the subsequent formulas, the expression $o / o$ is supposed to be equal to 1 .

Theorem 1. Let the function $\varphi$ satisfy conditions (1.1). Then equality (1.3) holds iff there exists such a number $\lambda \in \mathbb{R}$, for which the following equalities hold:

$$
\begin{gathered}
\lambda=\frac{\varphi(t+h)-2 \varphi(h)+\varphi(t-h)+\varphi(t)}{\varphi(t)}=\frac{\varphi(t-2 h)-2 \varphi(h)+\varphi(t-h)+\varphi(t)}{\varphi(t-h)}= \\
=\frac{2 \varphi(2 h)-\varphi(t-2 h)-\varphi(t)-\varphi(t-h)}{2 \varphi(h)-\varphi(t-h)}=\frac{2 \varphi(2 h)-\varphi(t-h)-\varphi(t+h)-\varphi(t)}{2 \varphi(h)-\varphi(t)}, \\
0 \leq t \leq h .
\end{gathered}
$$

P r o o f. By virtue of symmetry of the generalized $B$-spline w.r.t. the middle of the segment carrier, it is possible to think that $A_{1}=A_{4}$ and $A_{2}=A_{3}$.

Consider equality (1.3) as an equation w.r.t. the coefficients $A_{1}, A_{2}, A_{3}$ and $A_{4}$ on each segment $[0, h],[h, 2 h], \ldots,[5 h, 6 h]$. We obtain that

$$
\begin{gathered}
A_{1}=A_{4}=\frac{m(2 h)}{m(h)} \\
A_{2}=A_{3}=\frac{m(2 h)}{m(h)} \frac{[\varphi(t+h)-2 \varphi(h)+\varphi(t-h)+\varphi(t)]}{\varphi(t)}= \\
=\frac{m(2 h)}{m(h)} \frac{[\varphi(2 h-t)-2 \varphi(h)+\varphi(t-h)+\varphi(t)]}{\varphi(t-h)}= \\
=\frac{m(2 h)}{m(h)} \frac{[2 \varphi(2 h)-\varphi(t-2 h)-\varphi(t)-\varphi(t-h)]}{2 \varphi(h)-\varphi(t-h)}= \\
=\frac{m(2 h)}{m(h)} \frac{[2 \varphi(2 h)-\varphi(t-h)-\varphi(t+h)-\varphi(t)]}{2 \varphi(h)-\varphi(t)} .
\end{gathered}
$$

Examples. Give examples of three functions $\varphi$ that satisfy equalities (1.4). In the sequel for simplicity, we put $m(2 h)=m(h)$.

Example 1. Let $\varphi(x)=x^{2}$ (the parabolic splines). Then $A_{1}=A_{4}=1, A_{2}=A_{3}=3$ are the binomial coefficients from [8, formula 4.3.4].

Example 2. Let $\varphi(x)=\cosh (\beta x)-1(\beta>0)$ (the exponential splines corresponding to the linear differential operator of the third order with the form $\mathcal{L}_{3}=\mathcal{L}_{3}(D)=D\left(D^{2}-\beta^{2}\right)$, where $D$ is the differentiation symbol). Then $A_{1}=A_{4}=1, A_{2}=A_{3}=1+2 \cosh \beta h$. 
Example 3. Let $\varphi(x)=1-\cos \alpha x(\alpha>0)$, i.e., be the trigonometric splines corresponding to the linear differential operator of the third order with the form $\mathcal{L}_{3}=\mathcal{L}_{3}(D)=D\left(D^{2}+\alpha^{2}\right)$. Then $A_{1}=A_{4}=1, A_{2}=A_{3}=1+2 \cos \alpha h$.

In connection with the latter two examples, note the Author's work [12]. There the scaling relations are constructed for the $B$ - $\mathcal{L}$-splines (of an arbitrary order) in more generalized form than in (1.3).

\section{Generalized linear $B$-splines}

The scheme suggested for obtaining the two-scaled relations can be expanded onto the generalized linear $B$-splines.

Let the function $\varphi$ be given on the segment $[0,2 h]$ and satisfy the following conditions:

$$
\varphi \in C[0,2 h], \quad \varphi(0)=0 .
$$

The generalized linear $B$-spline is described by the formula

$$
B_{h, 1}(x)=m(h)\left\{\begin{array}{cl}
\varphi(x), & x \in[0, h], \\
\varphi(2 h-x), & x \in[h, 2 h], \\
0, & x \notin[0,2 h] .
\end{array}\right.
$$

Here, $m(h)>0$ is the normalizing multiplier. If to put $\varphi(x)=x$ and $m(h)=1 / h$, then formula (2.2) defines the normalized linear $B$-spline (see, for example [11]).

It is evident that $\operatorname{supp} B_{h, 1}=[0,2 h], B_{h, 1} \in C(\mathbb{R}), B_{h, 1}(2 h-x)=B_{h, 1}(x) \quad(x \in[0, h])$. Also, consider the function

$$
B_{2 h, 1}(x)=m(2 h)\left\{\begin{array}{cl}
\varphi(x), & x \in[0,2 h], \\
\varphi(4 h-x), & x \in[2 h, 4 h], \\
0, & x \notin[0,4 h]
\end{array}\right.
$$

that was obtained by formal substitution of the parameter $h$ by $2 h$ one in the function $B_{h, 1}$. We are interested in the question: for what $\varphi$ the equality holds

$$
B_{2 h, 1}(x)=C_{1} B_{h, 1}(x)+C_{2} B_{h, 1}(x-h)+C_{3} B_{h, 1}(x-2 h) \quad(x \in \mathbb{R}),
$$

where $C_{1}, C_{2}$, and $C_{3}$ are some real numbers?

Theorem 2. Let the function $\varphi$ satisfy conditions (2.1). Then equality (2.3) holds iff there exists such a number $\lambda \in \mathbb{R}$ for which the following equalities hold:

$$
\lambda=\frac{\varphi(t+h)-\varphi(h-t)}{\varphi(t)}=\frac{\varphi(2 h-t)-\varphi(t)}{\varphi(h-t)} \quad(0 \leq t \leq h) .
$$

P r o o f. It is similar to one of Theorem 1. Under this,

$$
\begin{gathered}
C_{1}=C_{3}=\frac{m(2 h)}{m(h)}, \\
C_{2}=\frac{m(2 h)}{m(h)} \frac{[\varphi(t+h)-\varphi(h-t)]}{\varphi(t)}=\frac{m(2 h)}{m(h)} \frac{[\varphi(2 h-t)-\varphi(t)]}{\varphi(h-t)} .
\end{gathered}
$$


Examples. As in the previous paragraph, it is possible to give examples of three functions $\varphi$ satisfying equalities (2.4). Again for simplicity, we put $m(2 h)=m(h)$.

Example 4. Let $\varphi(x)=x$ (the linear splines). Then $C_{1}=C_{3}=1, C_{2}=2$ are the binomial coefficients from [8, formula 4.3.4].

Example 5. Let $\varphi(x)=\sinh \beta x(\beta>0)$, i.e., be the exponential splines of the second order corresponding to the linear differential operator of the form $\mathcal{L}_{2}=\mathcal{L}_{2}(D)=D^{2}-\beta^{2}$. Then $C_{1}=C_{3}=1, C_{2}=2 \cosh \beta h$.

Example 6. Let $\varphi(x)=\sinh \alpha x(\alpha>0)$, i.e., be the trigonometric splines of the second order corresponding to the linear differential operator of the form $\mathcal{L}_{2}=\mathcal{L}_{2}(D)=D^{2}+\alpha^{2}$. Then $C_{1}=C_{3}=1, C_{2}=2 \cos \alpha h$.

\section{Conclusion}

It would be interesting to construct examples of other functions $\varphi$ satisfying relations (1.4) or (2.4). But it is not clear, how using only one function $\varphi$, it is possible to construct analogues of the polynomial $B$-splines of more high degrees, i.e., to derive formules of the type (1.2).

\section{REFERENCES}

1. Alberg J., Nilson E., Walsh J. Theory of splines and their applications. Moscow: Mir, 1972. 318 p. [in Russian]

2. Shevaldin V.T. Estimations from below of diameters of classes of source-represented functions // Trudy Steklov Math. Institute of RAS, 1989. Vol. 189. P. 185-201. [in Russian]

3. Rvachev V.A. Finite solutions of functional-differentional equations and their applications // Uspekhy Math. Nauk, 1990. Vol. 45, no. 1. P. 77-103. [in Russian]

4. Leontiev V.L. Orthogonal finite functions and numerical methods. Ulyanovsk: Ulyanovsk State University, 2003. 181 p. [in Russian]

5. Kvasov B.I. Methods for the iso-geometric approximation by splines. Moscow: Fizmatlit, 2006. 360 p. [in Russian]

6. Demyanovich Yu.K. Wavelet basis for $B_{\varphi}$-splines on a non-uniform mesh // Math. Modelling, 2006. Vol. 18, no. 10. P. 123-126. [in Russian]

7. Shevaldin V.T. Three-point scheme for approximation by local splines / Proceedings of International Summer Math. School by the name of S.B. Stechkin on the Theory of Functions. Tula: Tula State University, 2007. P. 151-156. [in Russian]

8. Chui Ch. Introduction into wavelets. Moscow: Mir, 2001. 412 p. [in Russian]

9. Subbotin Yu.N., Chernykh N.I. Construction of $W_{2}^{m}(\mathbb{R})$ wavelets and their approximative properties in various metrics // Proc. of Instit. of Math. and Mech. Ural Branch of RAS, 2005. Vol. 11, no. 2. P. 131-167. [in Russian]

10. Novikov I.Ya., Protasov V.Yu., Skopina M.A. Theory of wavelets. Moscow: Fizmatlit, 2005. 616 p. [in Russian]

11. Zavyalov Yu.S., Kvasov B.I., Miroshnichenko V.L. Spline-functions methods. Moscow: Nauka, 1980. 355 p. [in Russian]

12. Shevaldin V.T. Calibration relations for $B$ - $L$-splines. Modern problems of mathematics: Abstracts of 42nd Russian Youth Conference. Instit. of Math. and Mech. Ural Branch of RAS: Ekaterinburg, 2011. P. 151-153. [in Russian] 